\section{Familial Medullary Carcinoma of the Thyroid}

Medullary carcinoma of the thyroid is a tumour of the parafollicular or $\mathrm{C}$ cells, which produce calcitonin. It tends to occur in families, and is associated with phaeochromocytomas, parathyroid hyperplasia or adenomas, and occasionally with Cushing's syndrome. ${ }^{2}$ It has been classified ${ }^{3}$ as the multiple-endocrine-adenomatosis syndrome, type II, as distinct from the type I syndrome characterized by pituitary, parathyroid, pancreatic islet cell, and adrenal adenomas. Some patients with medullary carcinoma of the thyroid have a combination of multiple buccal and orbital neuromas, an unusual facies with thick lips, a marfanoid habitus, a proximal myopathy, and megacolon. ${ }^{4}$ Most cases with multiple neuromas have been sporadic, and this could prove to be an entity distinct from the multiple-endocrine-adenomatosis syndrome type II.

It should now be standard practice to investigate the families of patients with the medullary carcinoma of the thyroid syndrome: the disease is inherited as an autosomal dominant with a high degree of penetrance. ${ }^{5}$ Clinical evidence of the syndrome may be detected and may warrant surgical intervention. Biochemical tests should be performed to exclude the presence of a phaeochromocytoma before operating on the thyroid. In the familial syndrome bilateral phaeochromocytomas may be asymptomatic and appear to be non-functioning, but under conditions of stress such as a surgical operation a catastrophic release of catecholamines may occur. Routine screening tests such as the urinary excretion of hydroxymethoxymandelic acid (HMMA) may be negative, since small tumours have a faster turnover rate of catecholamines ${ }^{6}$ and may produce an increased excretion of free catecholamines in the urine without a corresponding increase in HMMA. Adrenal echograms or arteriograms or estimation of the catecholamine content of specimens obtained by venous catheterization may also provide evidence for the presence of the adrenal tumour.

Parathyroid hyperplasia, or less often adenoma formation, is part of the syndrome but does not always cause features of hyperparathyroidism with hypercalcaemia. It is uncertain whether the parathyroid hyperplasia is a response to the increased circulating calcitonin levels or to a genetically associated abnormality. Raised parathormone levels in the absence of abnormalities of circulating calcitonin were found in one kindred, ${ }^{7}$ which gives support to the latter possibility.

In the absence of clinically detectable thyroid enlargement radioisotope scanning of the thyroid may be helpful; calcification in the tumour or secondary deposits has been described. ${ }^{8}$ The most accurate screening test for the presence of medullary carcinoma is measurement of calcitonin in the peripheral circulation. ${ }^{9} \mathrm{~A}$ raised serum level of calcitonin in a patient with the familial syndrome with or without mucosal neuromas is an indication for total thyroidectomy after careful exclusion of a phaeochromocytoma. In a small proportion of cases the basal calcitonin level is normal, but a test using a calcium infusion to stimulate calcitonin secretion may produce an abnormality. In patients at risk who have normal basal calcitonin levels and a normal response to calcium it is probably wise to perform annual calcium infusion tests to detect disease at an early stage, and indeed two sisters at risk for hereditary medullary carcinoma who were tested in this way were found to have C-cell hyperplasia in the middle and upper portions of the lateral lobes of the thyroid. ${ }^{10}$ At that stage the process may be preinvasive, so that total thyroidectomy should prove to be curative. The increased numbers of $\mathrm{C}$-cells in the abnormal glands may be shown by routine haematoxylin and eosin staining as well as by more elaborate immunofluorescent or immunoenzymatic techniques. The multifocal nature of the bilateral hyperplasia correlates with the multicentric origin of the medullary carcinoma.

Calcitonin measurements are preferable to serum histaminase estimation ${ }^{11}$ in the diagnosis of medullary carcinoma of the thyroid. When provocative stimuli are required calcium infusions seem to be more reliable than glucagon or gastrin, which also stimulate the release of calcitonin. Furthermore glucagon is known to release catecholamines, which would prove dangerous in a patient with a phaeochromocytoma.

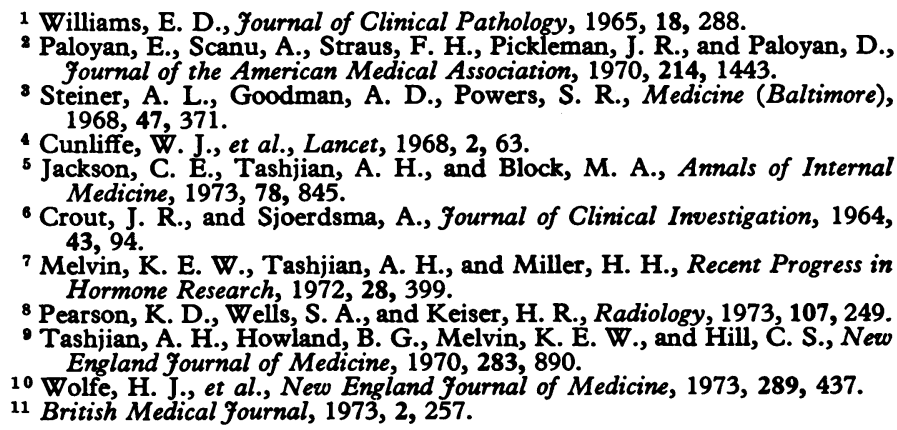

\section{Treatment of Genital Herpes}

Effective chemotherapy for virus infections is a challenge to biochemical ingenuity, since the complexity of the union between host and virus makes it difficult to inhibit the multiplication of the virus without at the same time damaging the host cells. One of the more elegant solutions is to incorporate a light-sensitive compound into the virus and then to irradiate the cells. The virus is made hypersensitive to light and is inactivated by the illumination. It is nearly 10 years since Wallis and Melnick ${ }^{1}$ suggested that this principle might be harnessed to the treatment of herpetic lesions in man, using a heterotricyclic dye such as acridine orange. A controlled trial using neutral red gave some success, ${ }^{2}$ and recently the method has been used in herpes vulvitis by Friedrich, ${ }^{3}$ Lefebure and McNellis, ${ }^{4}$ and a group including Wallis and Melnick themselves. ${ }^{5}$ This last study concerned 49 women aged 8 to 52 suffering from herpes genitalis. Twenty cases were described as primary and 28 as recurrent on serological grounds, the remaining one being unclassified. The duration of symptoms before treatment was in most cases less than 7 days. Local application of $0.1 \%$ proflavine was followed by irradiation with a 150-watt incandescent or fluorescent light for 10 minutes. The patients reported back in 24 hours and the results were assessed. Over half reported relief of pain within 2 hours, and most within 24 hours. Only 6 reported the degree of pain relief as poor, and even when the observed appearances were not much improved there was considerable subjective improvement. Kaufman et al. suggest that for virological reasons it would have been best to have given another treatment after 18 hours and that the use of monochromatic light may prove to be an advantage.

The evaluation of these results is difficult. Herpes genitalis is an unpleasant and troublesome condition, therapeutically somewhat intractable, and like all herpes infections apt to recur. It could be argued that any alleviation caused by the 
drug will be obvious because the results of leaving patients untreated are so unfavourable. In an earlier report ${ }^{6} 52$ out of Felber's 60 patients said his treatment gave better results than anything they had tried before. On the other hand 15 out of the 28 patients with recurrent disease in the present series reported pain relief as "immediate"-occurring in less than one hour-and it is difficult to see how the drug and the light could have worked so fast. Further, the proflavine or neutral red may have affected the associated secondary bacterial infection. This kind of uncertainty underlines the need for a controlled trial, and, as the authors admit, "this is not a wellcontrolled double-blind study, and the results do nothing more than suggest that a properly conducted study would be worthwhile undertaking". There are disadvantages: any vesicles have to be unroofed, an unpleasant procedure for both operator and patient. A more effective approach may prove to be the use of one of the drugs now known to be effective against herpes in other sites such as idoxuridine or cytosine arabinoside. Whatever the therapy adopted, two assertions can be made. The success of treating herpes infections at other sites has been such that it is unlikely that an effective therapy cannot be devised for genital herpes. On the other hand, it will only be by a controlled trial that the effectiveness, let alone the optimal dosage regimen, will be established.

\footnotetext{
1 Wallis, C., and Melnick, J. L., Photochemistry and Photobiology, 1966, 4, 159 Felber, T. D., Smith, E. B., Knox, J. M., Wallis, C., and Melnick, J. L.,

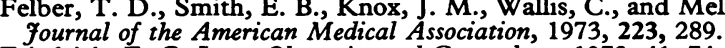

${ }^{3}$ Friedrich, E. G. Jun., Obstetrics and Gynecology, 1973, 41, 74.

Lefebure, E. B., and McNellis, E. E., fournal of the American Medical Association, 1973, 224, 1039.

${ }^{5}$ Kaufman, R. H., et al., American fournal of Obstetrics and Gynecology, $1973,117,1144$.

Fournal of the American Medical Association 1971, 217, 270.
}

\section{Haemophilus influenzae Infections}

Factors responsible for a decline in the incidence of infection with a particular agent can at times be clearly identifiedimprovements in standards of hygiene and immunization procedures, for example, have controlled typhoid, diphtheria, and polio. On the other hand an increase in the prevalence of an infection is generally more difficult to explain. Infections with Haemophilus influenzae have shown a considerable increase in the past three or four decades. ${ }^{1-3}$ These occur mainly in infancy and early childhood, and there has not been any marked change in the age group at greatest risk. Serious infections do occur in adults, however, as reported by Dr. Susannah Eykyn and her colleagues this week (p. 463).

Several types of encapsulated $H$. influenzae can be identified but only one, type $b$, is commonly responsible for clinical disease in man. The portal of entry is usually the respiratory tract, and the most frequent and serious forms of disease are meningitis and epiglottitis. Otitis media is also common, and less frequently pneumonia, septicaemia, septic arthritis, osteomylitis, and cellulitis can be caused by these organisms. $H$. influenzae has remained remarkably stable in respect of its sensitivity to antibiotics; chloramphenicol, ampicillin, and the tetracyclines are still generally effective, but recently there have been a few reports of organism resistant to ampicillin and chloramphenicol. However, one factor in the seriousness of the infection in some sites is that the early clinical signs can be mild and non-specific, and this often results in a delay in treatment.

$H$. influenzae meningitis is one of the major current concerns.
Recent estimates ${ }^{4}$ from the U.S.A. suggest that there are probably over 10,000 cases a year with 800 deaths, a mortality rate of $0 \cdot 4 / 100,000$ of the total population and 3.8/100,000 in children less than 5 years of age. Also of great concern is the high incidence (nearly $30 \%$ ) of residual brain damage in the survivors--and this estimate does not include children with minor degrees of impaired learning ability, who may add another $10 \%$ to the incidence of morbidity.

The overall risk of meningitis in children under 5 is three times that ${ }^{2}$ in 1942-50. In a study of a population of 360,000 in North Carolina the number of cases has risen ${ }^{5}$ from nine per year in 1966 to 27 per year in 1971, and in children under 5 the attack rate was three times greater in negroes than in white children.

Statistics like these have been a stimulus for research into preventive measures. There have been two approaches in the development of a vaccine. In the first a type-specific antigen has been prepared from the external polyribophosphate capsule, thought to be critical in the pathogenicity of $H$. influenzae. Experiments on animals have shown ${ }^{6}$ that antibody to this antigen in antiserum is protective and anti-type $b$ antibodies in man have also been found ${ }^{7}$ effective. A vaccine composed of the capsular polysaccharide has been subjected ${ }^{8}{ }^{9}$ to trials in human volunteers which showed that antibody responses were both dose- and age-dependent. In children under 12 months of age, however, antibody responses were most unsatisfactory -and this is the age at which the attack rates are highest. A similar phenomenon of age-related antibody response has also been noted ${ }^{1011}$ in natural infection, when little or no antibody was detected in infants. The reasons for these findings are at present unclear and may not necessarily be due to a complete absence of an immune response; indeed using a more sensitive test to measure antibody a response to this type of vaccine has been detected ${ }^{12}$ in 2- and 3-month-old infants.

The second, indirect approach to immunization is of considerable theoretical interest. It concerns antigens from normal bacterial flora which crossreact because of a component they share with the capsular polysaccharide of $H$. influenzae type b. Several such bacteria have been identified, ${ }^{12}$ including strains of Escherichia coli and other non-pathogenic enteric bacteria. It may be possible ${ }^{15}$ to induce immunity by neonatal feeding with such non-pathogenic enteric bacteria.

The implications for vaccine development are clearly of great importance, but there are many practical problems still to be faced. For example, who is to be immunized and at what age? Answers to such questions are not, however, going to be required until these experimental vaccines have been proved to be of practical value.

1 Michaels, R. H., New England Fournal of Medicine, 1971, 285, 666.

2 Smith, E. W. P., and Haynes, R. E., Pediatrics, 1972, 50, 723

Turk, D. C., and May, J. R., Haemophilus Influenzae, p. 27. London, English Universities Press, Ltd., 1967.

Mortimer, E. A., Pediatrics, 1973, 52, 633.

S Parke, J. C., Schneerson, R., and Robbins, J. B., Fournal of Pediatrics $1972,81,765$.

Alexander, H. E., Heidelberger, M., and Leidy, G., Yale fournal of Biology and Medicine, 1944, 16, 425.

7 Schneerson, R., Rodrigues, L. P., Parke, J. C., and Robbins, J. B.,

Fournal of Immunology, 1971, 107, 1081.
8 Anderson, P., Peter, G., Johnston, R. B., Wetterlow, L. H., and Smith, D. H., Fournal of Clinical Investigation, 1972, 51, 39.

Smith, D. H., Peter, G., Ingram, D. L., Harding, A. L., and Anderson, P., Pediatrics, 1973, 52, 637.

${ }^{10}$ Peter, G. S., Greenfield, S., Howie, V. M., and Smith, D. H., Pediatric Research, 1971, 5, 401.

11 Norden, C. W., Melish, M., Overall, J. C., and Baum, J., fournal of Pediatrics, 1972, 80, 209.

12 Robbins, J. B., Parke, J. C., Schneerson, R., and Whisant, J., Pediatric Research, 1973, 7, 103.

13 Bradshaw, M. W., Schneerson, R., Parke, J. C., and Robbins, J. B., Lancet, 1971, 1, 1095.

14 Robbins, J. B., Annals of Internal Medicine, 1973, 78, 259.

15 Myerowitz, R. L., Handzel, Z. T., Schneerson, R., and Robbins, J. B., Infection and Immunity, 1973, 7, 137. 